\title{
How Do People Manage Interruptions in Complex Decision Making Tasks?
}

\author{
Sheryl L. Miller \\ Human Factors and Applied Cognitive Psychology \\ George Mason University \\ Fairfax, VA 22030-4444 USA \\ +17039931207 \\ smillerb@osf1.gmu.edu
}

\begin{abstract}
Interruption is a non-trivial part of complex work, in that interruptions frequently contain useful information. When choosing how to manage an interruption, this potential usefulness must be considered along with potential disruption to the ongoing task. This paper proposes two experiments that investigate the strategies that people use to manage interruptions in a computer-based, team decision making task.
\end{abstract}

\section{Keywords}

Interruption, decision making, strategy

\section{INTRODUCTION}

Understanding the role of interruptions in complex tasks is of increasing importance. A person can encounter interruptions in any setting (the car, working on a computer, or at the beach) due to intrusions from pagers, cellular phones, and instant messaging. Increasingly, these are not mere intrusions, but information-rich events that draw a person's full attention. For instance, pagers no longer contain only 7-digit phone numbers. Instead, they can contain many lines of text, internet updates, and even graphics or animation.

It is generally agreed that interruption is disruptive to primary task performance $[1,7]$. However, characteristics of interruptions have not been adequately manipulated, nor have effects of interruptions been adequately evaluated in complex settings where the interruption itself provides information crucial to ongoing performance. Two experiments will be proposed to determine how the timing and relative importance of task relevant interruptions interact with cognition to affect performance. Given what we know about human memory and attention, what type of interruptions are people able to most effectively manage?

The literature says very little about how people manage interruptions. Instead, it focuses on the degree of disruption that occurs in the primary task. (However, in many task domains, particularly complex ones where interruptions are not trivial elements of the primary task, task accuracy is also very important.) Generally, time to resume the primary task after being interrupted degrades, because the primary task resumption point is forgotten, due to factors like similarity between the primary task and the interruption event [1]. Previous work offers mixed evidence about whether or not people are able to improve resumption time for the primary task by controlling when they switch attention to the interruption [e.g., 1, 3, 7]. The proposed research extends previous work by asking: Are people able to strategically manage interruptions in complex task settings?

\section{PROPOSED WORK}

Task Domain

The experiments proposed here will use the Team Argus system [5]. Participants will classify a series of aircraft on a radar screen in terms of their threat and select an appropriate course of action for each. This will be accomplished using a set of complicated rules, including a multiplicative decision calculation. Each experimental participant will play the role of a member of a simulated three-person team. He or she will behave as though geographically separated from the rest of the "team," using only electronic communication. Each participant will expect to receive information cues (e.g., aircraft altitude) from the other members in order to be fully informed [2] about the environment and make optimal decisions.

The researcher, not the participant, will control communication by defining the frequency, timing, and content of messages. Intermittently, each participant will be interrupted with a message from a simulated teammate, containing information (to be used immediately or in the future, see Figure 1). Good performance will be based on correctly remembering the resumption point of the primary task and correctly remembering the information contained in an earlier interruption when it is needed. Thus, the Team Argus decision making task can be viewed as a "keeping track" task [6], in which a participants must keep track of information for future decision making, as well as keeping track of the current task. 


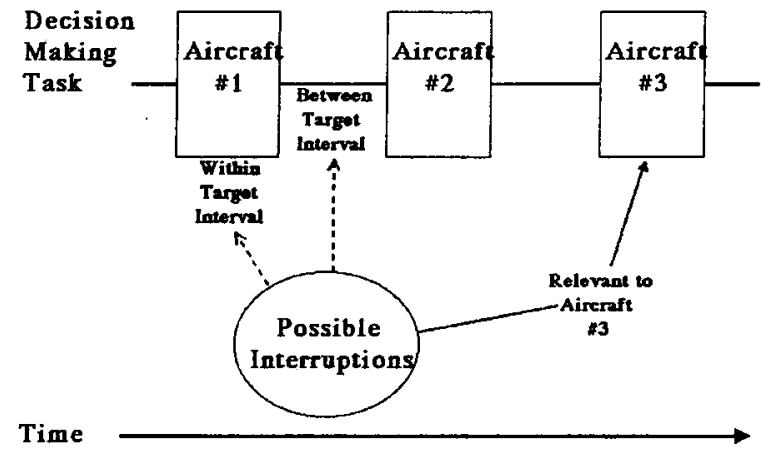

Figure 1. An example of interruptions that contain information relevant to a future decision.

\section{Experiment 1}

The first experiment will address whether people develop different strategies for managing interruptions while they are monitoring the radar scope, given the relative benefits of different interruptions. Will people react differently to interruptions that contain highly weighted decision cues than they do to interruptions that contain less important (low weighted) decision cues? It is expected that people will change the way that they process an interruption so they are more likely to remember highly weighted information in the future. Failure to remember important information will have a significant impact on task accuracy and time-on-task in future decision making. Therefore, people are expected to spend more time attending to an interruption in order to encode highly weighted task information than lower weighted task information.

It is expected that people's sensitivity to information importance will be mediated by two variables, the information maintenance period (between the interruption and the use of the information contained in the interruption) and working memory capacity (as determined by a pretest). The information contained in the interruption will degrade as the time between the interruption and information use increases. More so, this decline in memory for information should be a function of the amount of intervening activity (i.e., decision events) during the period. Increased intervening activity will lead to participant strategy changes that optimize memory for important information. It is also expected that participants with a high working memory capacity will be able to better maintain this information, and therefore, their strategies will be different from participants with low working memory capacity.

\section{Experiment 2}

The second experiment will compare the effects of interruptions (like those studied in Experiment 1) during cognitively non-intensive periods (monitoring the radar scope, i.e., Between Target Interval) and cognitively intensive periods (decision-making calculation, i.e., Within
Target Interval). Do people change their strategies for managing interruptions during periods of different cognitive intensity? It is expected that when people are interrupted while cognitively busy, they will strategically delay attending to the interruption so as to better encode and rehearse the resumption point of the primary task.

\section{CONCLUSION}

Past research of interruptions has not considered a task of sufficient complexity, in which time and accuracy are critical performance measures and interruptions are necessary and task relevant. How will participants strategize to manage interruptions to their benefit during the complex, computer-based Team Argus decision task? Given certain task conditions, it is expected that they will delay attending to the interruption and/or increase the length of an interruption so as to optimize overall task performance. Understanding the strategic shifts made by people who must deal with interruptions on an ongoing basis is important to predicting when performance will degrade and to predicting overall task success.

\section{ACKNOWLEDGMENTS}

The author would like to thank Deborah Boehm-Davis, Erik Altmann, Leonard Adelman, and Gregory Trafton for their comments on the proposed work and earlier drafts of this paper.

\section{REFERENCES}

1. Gillie, T., \& Broadbent, D. (1989). What makes interruptions disruptive? A study of length, similarity, and complexity. Psychological Research, 50, 243-250.

2. Hollenbeck, J. R., Ilgen, D. R., Sego, D. J., Hedlunc, J., \& et al. Mutlilevel theory of team decision making: Decision performance in teams incorporating distributed expertise. Journal of Applied Psychology, 80, 292-316.

3. McFarlane, D. C. (1998). Interruption of people in human-computer interaction. Doctoral dissertation, George Washington University, Washington, DC.

4. Miller, S. L., Adelman, L., Henderson, E. D., Schoelles, M., \& Yeo, C. (2000). Team DecisionMaking Strategies: Implications for Designing the Interface in Complex Tasks. In Proceedings of the $44^{\text {th }}$ Annual Human Factors and Ergonomics Society. Santa Monica, CA: Human Factors and Ergonomics Society.

5. Schoelles, M. \& Gray W. D. (1998). Argus, A System for Varying Cognitive Workload. In Proceedings of the Human Factors and Ergonomics Society 42 $2^{\text {nd }}$ Annual Meeting. Santa Monica: Human Factors and Ergonomics Society.

6. Venturino, M. (1997). Interference and information organization in keeping track of continually changing information. Human Factors, 39, 532-539.

7. Zijlstra, F. R. H., Roe, R. A., Leonora, A. B., \& Krediet, I. (1999). Temporal Factors in mental work: Effects of interrupted activities. Journal of Occupational and Organizational Design. 72, 163-185. 